Appl. Set-Valued Anal. Optim. 1 (2019), No. 1, pp. 29-38

Available online at http://asvao.biemdas.com

https://doi.org/10.23952/asvao.1.2019.1.03

\title{
WELL-POSEDNESS FOR BILEVEL VECTOR EQUILIBRIUM PROBLEMS
}

\author{
XINGXING JU ${ }^{1, *}$, SUHEL AHMAD KHAN ${ }^{2}$ \\ ${ }^{1}$ School of Mathematics and Statistics, Southwest University, Chongqing 400715, China \\ ${ }^{2}$ Department of Mathematics, BITS-Pilani, Dubai Campus, P.O.Box 345055 Dubai, United Arab Emirates
}

\begin{abstract}
In this paper, we study the well-posedness for bilevel vector equilibrium problems (BVEP) and propose a generalized well-posed concept for the BVEP in Hausdorff topological vector spaces. We also discuss the relationship between the solution set and the approximating solution set for the BVEP. Finally, we establish a metric characterization for the generalized wellposedness in terms of the Kuratowski measure of the non-compactness of the approximating solution set.

Keywords. Bilevel vector equilibrium problems; Well-posedness; Upper (lower) semicontinuous; Approximating solution sequence.
\end{abstract}

2010 Mathematics Subject Classification. 49K40, 90C33.

\section{INTRODUCTION}

Well-posedness is one of the most important and interesting topics in the fields of optimization theory. Well-posedness means the existence and continuous dependence of optimal solutions and optimal values on perturbed date; see [1]. Tykhonov [2] introduced the notion of well-posedness of minimization problems, which means the existence of a minimizer and the convergence of a subsequence of each approximating sequence to a solution. In 1966, Levitin and Polyak [3] introduced a new notion of the well-posedness that strengthens the Tykhonov's concept as it requires the convergence to the optimal solution of each sequence, which belongs to a larger set of minimizing sequences. Up to now, the concept of well-posedness has been generalized to several related problems, such as, vector optimization problems [4, 5], vector variational inequalities [6], Nash equilibrium problems [7, 8] and vector equilibrium problems $[9,10,11,12]$ and the references therein. In the filed of well-posedness, it is very crucial to introduce a suitable approximating solution sequence related with iterative algorithms for the corresponding problems. Various aspects, such as, optimality conditions, stability and convergence of approximation solutions and approximating solution sequence, were studied; see, e.g., [13, 14] and the reference therein.

Recently, Moudafi [15] introduced a class of bilevel monotone equilibrium problem that consists of finding $\bar{x} \in S_{F}$ such that

$$
H(\bar{x}, y) \geq 0, \forall y \in S_{F},
$$

\footnotetext{
${ }^{*}$ Corresponding author.

E-mail addresses: juxxmath@163.com (X. Ju), khan.math@ gmail.com (S.A. Khan).

Received January 15, 2019; Accepted March 20, 2019.
}

(C)2019 Applied Set-Valued Analysis and Optimization 
where $S_{F}$ is the solution set of the following equilibrium problem that consists of finding $u \in K$ such that

$$
F(u, z) \geq 0, \forall z \in K
$$

where $K$ is a nonempty closed convex subset of a Hilbert space and $H, F: K \times K \rightarrow R$ are two functions. Moudafi pointed out that the BMEP includes bilevel variational inequalities, hierarchical optimization and optimization with equilibrium constraints as special cases and proposed a simple proximal method to solve the BMEP. Subsequently, the existence of solutions, well-posedness and algorithms for bilevel monotone equilibrium problems and bilevel variational inequalities have been extensively studied; see, e.g., [16, 17] and the references therein. In 2014, Anh, Khanh and Van [18] considered the Levitin-Polyak (LP) well-posedness of bilevel equilibrium problems with equilibrium constraints and bilevel optimization problems with equilibrium constraints. They introduced the relaxed level closedness and used it together with the pseudocontinuity assumptions to establish sufficient conditions for well-posedness and unique well-posednedss. In 2013, Chen, Cho and Wan [19] introduced and investigated some topological properties of solutions for lower level mixed equilibrium problems and bilevel mixed equilibrium problems in reflexive Banach space which consists of finding $x \in S_{\Psi, B, \psi}$ such that

$$
\Phi(x, y)+\langle A x, y-x\rangle+\phi(y, x)-\phi(x, x) \geq 0, \forall y \in S_{\Psi, B, \psi},
$$

where $S_{\Psi, B, \psi}$ is the solution set of the lower level mixed equilibrium problem: find $y^{*} \in K$ such that

$$
\Psi\left(y^{*}, z\right)+\left\langle B y^{*}, z-y^{*}\right\rangle+\psi\left(z, y^{*}\right)-\psi\left(y^{*}, y^{*}\right) \geq 0, \forall z \in K,
$$

where $X$ is a real Banach space with its dual space $X^{*}, K$ is a nonempty convex subset of $X, \Phi, \Psi, \phi, \psi$ : $K \times K \rightarrow R \cup\{+\infty\}$ are four functions and $A, B: X \rightarrow X^{*}$ are two vector-valued mappings. They proved, under suitable conditions, the well-posedness and generalized well-posedness are equivalent to the uniqueness and existence of its solutions. Khanh, Plubtieng and Sombut [20] introduced several types of LP well-posedness for bilevel vector equilibrium and optimization problems with equilibrium constraints. Based on criteria and characterizations for these types of LP well-posedness, they argued on diameters and Kuratowski's, Hausdorff's, or Istratescu's measures of non-compactness of approximate solution sets under suitable conditions, and proved the LP well-posedness for bilevel vector equilibrium and optimization problems with equilibrium constraints. In 2017, Chadli, Ansari and Al-Homidan [21] introduced the bilevel vector equilibrium problems and established some existence results for them by using a vector Tykhonov-type regularization procedure and existence results for mixed vector equilibrium problems. They also studied the uniqueness of solutions of the auxiliary vector equilibrium problems. They presented an algorithm for computing the approximating solutions and studied the strong convergence of the proposed algorithm. In, Anh and Hung [22] introduced the bilevel vector equilibrium problems and studied the stability conditions, such as, the Hausdorff upper semicontinuity, the Hausdorff lower semicontinuity, the outer-continuity and the outer-poenness of solutions for such problems. In 2018, Anh and Hung [23] further considered strong bilevel vector equilibrium problems and introduced the concepts of LP well-posedness and LP well posedness in the generalized sense for such problems. Note that the well-posedness of the bilevel vector equilibrium problems was studied under the existence of solution assumptions in the literature. However, it is not easy to ensure the well-posedness and existence of solution of the bilevel vector equilibrium problems simultaneously. So, it is of interest to study the well-posedness of bilevel vector equilibrium problems under the compatible conditions which ensure the existence of solutions. 
In this paper, motivated and inspired by the above results, we introduce and investigate the wellposedness for bilevel vector equilibrium problems. We introduce the well-posedness and generalize the well-posedness for the bilevel vector equilibrium problems. Under mild conditions, we obtain existence approximating solution theorems. Metric characterizations of the generalized well-posedness of the bilevel vector equilibrium problems are established by the Kuratowski measure of non-compactness of the approximating solution set.

This paper is organized as follows. In Section 2, we recall some basic definitions. In Section 3, an existence theorem in the exact settings and approximating settings are established. In Section 4, we establish a metric characterization for the generalized well-posedness of the bilevel vector equilibrium problems by using the Kuratowski measure of non-compactness of the approximating solution set. Several examples are given for the illustration of our main results.

\section{PRELIMINARIES}

Let $X$ and $Z$ be Hausdorff topological vector spaces. Throughout this paper, we assume that $K$ is a nonempty, closed and convex subset of $X$, and $C \subset Z$ is a proper, closed, convex and pointed cone with $\operatorname{int} C \neq \emptyset$, where $\operatorname{int} C$ is the interior of $C$. Let $F, H: K \times K \rightarrow Z$ be two vector-valued bifunctions and $\omega \in \operatorname{int} C$.

Now, we recall the following bilevel vector equilibrium problem (BVEP) [21]: find $\bar{x} \in \Delta$ such that

$$
H(\bar{x}, y) \notin-\operatorname{int} C, \quad \forall y \in \Delta,
$$

where $\Delta$ is the set of solutions of the lower level vector equilibrium problem: find $\bar{z} \in K$ such that

$$
F(\bar{z}, z) \notin-\operatorname{int} C, \quad \forall z \in K .
$$

In many practical problems, we usually obtain the approximated solution of the BVEP instead of the precise solution. So, it is necessary to consider the approximation solutions of the BVEP. Let $\varepsilon \in[0,+\infty[$ be the tolerance error. We consider the following $\varepsilon$-bilevel vector equilibrium problem ( $\varepsilon$-BVEP): find $\bar{x} \in K$ such that

$$
\begin{aligned}
& H(\bar{x}, y)+\varepsilon \omega \notin-\operatorname{int} C, \quad \forall y \in \Delta, \\
& F(\bar{x}, z)+\varepsilon \omega \notin-\operatorname{int} C, \quad \forall z \in K .
\end{aligned}
$$

The solution of the $\varepsilon$-BVEP is also called the $\varepsilon$-solution of the BVEP. We denote by $\Gamma(\varepsilon)$ and $\Gamma$ the set of $\varepsilon$-solutions and the set of solutions of the BVEP, respectively. Particularly, we denote

$$
\Delta(\varepsilon)=\{\bar{x} \in K: F(\bar{x}, z)+\varepsilon \omega \notin-\operatorname{int} C, \forall z \in K\} .
$$

Definition 2.1. A sequence $\left(x_{n}\right)_{n \in N} \subseteq K$ is said to be an approximating solution sequence for the BVEP if and only if there exists a positive sequence with $\left(\varepsilon_{n}\right)_{n \in N}$ with $0<\varepsilon_{n} \rightarrow 0$ as $n \rightarrow+\infty$ such that

$$
H\left(x_{n}, y\right)+\varepsilon_{n} \omega \notin-\operatorname{int} C, \forall y \in \Delta,
$$

and

$$
F\left(x_{n}, z\right)+\varepsilon_{n} \omega \notin-\mathrm{int} C, \forall z \in K .
$$

Definition 2.2. The BVEP is said to be well-posed if the following conditions hold:

(i) $\Gamma$ is a singleton set, i.e., $\Gamma=\{\bar{x}\}$; 
(ii) every approximating sequence $\left(x_{n}\right)_{n \in N}$ of (BVEP) converges to $\bar{x}$.

Definition 2.3. The BVEP is said to be generalized well-posed if $\Gamma \neq \emptyset$ and every approximating sequence $\left(x_{n}\right)_{n \in N}$ of the BVEP has a subsequence which converges to some point in $\Gamma$.

Remark 2.1. From Definition 2.2 and Definition 2.3, we know that if The BVEP is well-posed or generalized well-posed, then $\Gamma$ is compact.

Definition 2.4. [24] A vector-valued function $G: X \rightarrow Z$ is said to be $C$-lower semicontinuous on $X$ if it satisfies any one of the following conditions:

(i) for all $a \in Z, G^{-1}(a+\operatorname{int} C)$ is open;

(ii) for each $x_{0} \in X$ and any open neighborhood $V$ of $G\left(x_{0}\right)$, there exists an open neighborhood $U$ of $x_{0}$ such that $G(x) \in V+C$ for all $x \in U$;

(iii) for each $x_{0} \in X$ and any $d \in \operatorname{int} C$, there exists an open neighborhood $U$ of $x_{0}$ such that $G(x) \in$ $G\left(x_{0}\right)-d+\operatorname{int} C$ for all $x \in U$.

$G$ is also said to be $C$-upper semicontinuous on $X$ if $-G$ is $C$-lower semicontinuous on $X$.

Definition 2.5. [25] The vector-valued bifunction $H: K \times K \rightarrow Z$ is said to be strictly $C$-weakly quasiconcave in the first argument if, for each $x_{1}, x_{2} \in K$ with $x_{1} \neq x_{2}$, for any $\lambda \in(0,1)$ and $y \in K$, we have

$$
H\left(x_{1}, y\right) \notin-\operatorname{int} C, H\left(x_{2}, y\right) \notin-\operatorname{int} C \Rightarrow H\left(\lambda x_{1}+(1-\lambda) x_{2}, y\right) \notin-C .
$$

Lemma 2.1. For each $x \in C, y \notin-i n t C$ implies $x+y \notin-i n t C$.

\section{THE EXISTENCE OF APPROXIMATING SOLUTIONS FOR THE BVEP}

The existence of solutions to the BVEP has been studied in [21]. We assume that $\Delta \neq \emptyset$ and $\Gamma \neq$ $\emptyset$. In this section, the existence of approximating solutions to the BVEP is established under suitable conditions. Furthermore, the convexity and closedness of the sets of approximated solutions of the BVEP and its lower level problem are derived.

Lemma 3.1. [26] Let $X$ and $Z$ be Hausdorff topological vector spaces. Assume that $K$ is a nonempty, closed and convex subset of $X$. Let $F: K \times K \rightarrow Z$ be two vector-valued bifunctions. Assume that, for each $y \in K, x \mapsto F(x, y)$ is strictly $C$-weakly quasiconcave on $K$ in the first argument. Then the following statements hold:

(1) $\Delta$ is convex and close;

(2) for each $\varepsilon>0, \Delta(\varepsilon)$ is nonempty, closed, convex;

(3) $\Delta=\bigcap_{\varepsilon>0} \Delta(\varepsilon)$.

Theorem 3.1. Let $X$ and $Z$ be a Hausdorff topological vector spaces, and let $K$ be a nonempty, closed and convex subset of $X$. Let $F, H: K \times K \rightarrow Z$ be two vector-valued bifunctions. Suppose that

(i) for each $y \in K, x \mapsto H(x, y)$ is strictly $C$-weakly quasiconcave on $K$ in the first argument;

(ii) for each $y \in K, x \mapsto F(x, y)$ is strictly $C$-weakly quasiconcave on $K$ in the first argument.

Then the following statements hold:

(1) $\Gamma$ is closed and convex; 
(2) for each $\varepsilon>0, \Gamma(\varepsilon)$ is nonempty, closed, convex.

Proof. (1) First, we show $\Gamma$ is closed. Suppose that $\left(x_{n}\right)_{n \in N} \subset \Gamma$ and $x_{n} \rightarrow \bar{x}$. Then we have

$$
H\left(x_{n}, y\right) \notin-\operatorname{int} C, \forall y \in \Delta,
$$

where

$$
\Delta=\left\{x_{n} \in K: F\left(x_{n}, z\right) \notin-\operatorname{int} C, \forall z \in K\right\} .
$$

It needs to show that $H(\bar{x}, y) \notin-\operatorname{int} C$ for all $y \in \Delta$. Assume that there exists $\hat{y} \in \Delta$ such that $H(\bar{x}, \hat{y}) \in$ $-\operatorname{int} C$. Then, there is $a \in \operatorname{int} C$ such that

$$
H(\bar{x}, \hat{y})+a \in-\operatorname{int} C .
$$

Since $H$ is $C$-upper semicontinuous on $K$ in the first argument, there exists an open neighborhood $U$ of $\bar{x}$ such that

$$
H\left(x_{n}, \hat{y}\right) \in H(\bar{x}, \hat{y})+a-\operatorname{int} C, \quad \forall x_{n} \in U .
$$

From (3.2), we deduce that $H\left(x_{n}, \hat{y}\right) \in-\operatorname{int} C$, which contradicts (3.1). Hence,

$$
H(\bar{x}, y) \notin-\operatorname{int} C, \forall y \in \Delta,
$$

where

$$
\Delta=\{\bar{z} \in K: F(\bar{z}, z) \notin-\text { int } C, \forall z \in K\} .
$$

Therefore, $\bar{x} \in \Gamma$.

We now show the convexity of $\Gamma$. If $\Gamma$ is a singleton, then it is trivial. Suppose that $x_{1}, x_{2} \in \Gamma, x_{1} \neq x_{2}$. Then we have

$$
H\left(x_{1}, y\right) \notin-\operatorname{int} C \text { and } H\left(x_{2}, y\right) \notin-\operatorname{int} C \text {. }
$$

From condition (ii) and Lemma 3.1, we know that $\Delta$ is convex. By the convexity of $\Delta$,

$$
t x_{1}+(1-t) x_{2} \in \Delta, \forall t \in(0,1) .
$$

By the strictly $C$-weakly quasiconcavity of $H(x, y)$ in the first argument, we obtain $H\left(t x_{1}+(1-t) x_{2}, y\right) \notin$ $-C$, which implies that

$$
H\left(t x_{1}+(1-t) x_{2}, y\right) \notin-\operatorname{int} C, \forall y \in \Delta,
$$

where

$$
\Delta=\{\bar{z} \in K: F(\bar{z}, z) \notin-\operatorname{int} C, \forall z \in K\} .
$$

Hence, $t z_{1}+(1-t) z_{2} \in \Gamma$, i.e., $\Gamma$ is a convex set.

(2) Since $\Gamma \neq \emptyset$, we have $\exists x \in K$ such that $H(x, y)+\varepsilon \omega \notin-\operatorname{int} C$ for all $y \in \Delta$ and $F(x, z)+\varepsilon \omega \notin-\operatorname{int} C$ for all $z \in K$. Considering $\omega \in \operatorname{int} C \subset C$, we have $\varepsilon \omega \in \operatorname{int} C \subset C$. Moreover, by Lemma 2.1, we obtain

$$
H(x, y)+\varepsilon \omega \notin-\operatorname{int} C, \forall y \in \Delta
$$

and

$$
F(x, z)+\varepsilon \omega \notin-\operatorname{int} C, \forall z \in K .
$$

Hence, $\Gamma(\varepsilon) \neq \emptyset$.

Next, we show that $\Gamma(\varepsilon)$ is closed. Letting $\left(x_{n}\right)_{n \in N} \subset \Gamma(\varepsilon)$ and $x_{n} \rightarrow \bar{x}$, we have

$$
H\left(x_{n}, y\right)+\varepsilon \omega \notin-\operatorname{int} C, \forall y \in \Delta
$$


and

$$
F\left(x_{n}, z\right)+\varepsilon \omega \notin-\operatorname{int} C, \forall z \in K \text {. }
$$

We need to prove that

$$
H(\bar{x}, y)+\varepsilon \omega \notin-\operatorname{int} C, \quad \forall y \in \Delta .
$$

Assume by contradiction that there exists $\bar{y} \in K$ such that $H(\bar{x}, \bar{y})+\varepsilon \omega \notin-\operatorname{int} C$ for all $x_{n} \in U$. Then, there is $a \in \operatorname{int} C$ such that

$$
H(\bar{x}, \bar{y})+\varepsilon \omega+a \notin-\operatorname{int} C .
$$

Since $H$ is $C$-upper semicontinuous on $K$ in the first argument, we see that there exists an open neighborhood $U$ of $\bar{x}$ such that

$$
H\left(x_{n}, \bar{y}\right) \in H(\bar{x}, \bar{y})+a-\operatorname{int} C,
$$

which implies that

$$
H\left(x_{n}, \bar{y}\right)+\varepsilon \omega \in H(\bar{x}, \bar{y})+\varepsilon \omega+a-\operatorname{int} C .
$$

By relation (3.6), we deduce that

$$
H\left(x_{n}, \bar{y}\right)+\varepsilon \omega \in-\operatorname{int} C,
$$

which contradicts (3.5). Hence,

$$
H(\bar{x}, y)+\varepsilon \omega \notin-\operatorname{int} C, \forall y \in \Delta,
$$

and

$$
F(\bar{x}, y)+\varepsilon \omega \notin-\operatorname{int} C, \forall z \in K .
$$

Therefore, $\bar{x} \in \Gamma(\varepsilon)$. The proof of the convexity of $\Gamma(\varepsilon)$ is similar to that of the convexity of $\Gamma$. This completes the proof.

Proposition 3.1. Let $X$ and $Z$ be Hausdorff topological vector spaces, $K$ be a nonempty, closed and convex subset of $X$. Let $F, H: K \times K \rightarrow Z$ be two vector-valued bifunctions. Suppose $\Gamma \neq \emptyset$, then

$$
\Gamma=\bigcap_{\varepsilon>0} \Gamma(\varepsilon)
$$

Proof. First, we show that

$$
\Gamma\left(\varepsilon_{1}\right) \subset \Gamma\left(\varepsilon_{2}\right), \quad \forall \varepsilon_{2}>\varepsilon_{1} \geq 0 .
$$

Suppose that $\bar{x} \in \Gamma\left(\varepsilon_{1}\right)$. Then we have

$$
H(\bar{x}, y)+\varepsilon_{1} \omega \notin-\operatorname{int} C, \forall y \in \Delta
$$

and

$$
F(\bar{x}, z)+\varepsilon_{1} \omega \notin-\operatorname{int} C, \forall z \in K .
$$

According to $\varepsilon_{2}-\varepsilon_{1}>0, \omega \in \operatorname{int} C$ and Lemma 2.1, we obtain

$$
H(\bar{x}, y)+\varepsilon_{1} \omega+\left(\varepsilon_{2}-\varepsilon_{1}\right) \omega \notin-\operatorname{int} C, \forall y \in \Delta
$$

and

$$
F(\bar{x}, z)+\varepsilon_{1} \omega+\left(\varepsilon_{2}-\varepsilon_{1}\right) \omega \notin-\operatorname{int} C, \forall z \in K .
$$


Hence,

$$
H(\bar{x}, y)+\varepsilon_{2} \omega \notin-\operatorname{int} C
$$

and

$$
F(\bar{x}, z)+\varepsilon_{2} \omega \notin-\operatorname{int} C,
$$

which implies that $\bar{x} \in \Gamma\left(\varepsilon_{2}\right)$. Therefore,

$$
\Gamma\left(\varepsilon_{1}\right) \subset \Gamma\left(\varepsilon_{2}\right) .
$$

On letting $\varepsilon_{1}=0, \varepsilon_{2}=\varepsilon>0$, we have $\Gamma \subset \Gamma(\varepsilon)$. Consequently,

$$
\Gamma \subset \bigcap_{\varepsilon>0} \Gamma(\varepsilon) .
$$

On the other hand, if $0<\varepsilon_{1}<\varepsilon_{2}$, we have $\Gamma\left(\varepsilon_{1}\right) \subset \Gamma\left(\varepsilon_{2}\right)$. It follows that

$$
\bigcap_{\varepsilon>0} \Gamma(\varepsilon)=\lim _{\varepsilon \rightarrow 0} \Gamma(\varepsilon)
$$

that is,

$$
\bigcap_{\varepsilon>0} \Gamma(\varepsilon)=\{x \in K: H(x, y) \notin-\operatorname{int} C, \forall y \in \Delta, F(x, z) \notin-\operatorname{int} C, \forall z \in K\} .
$$

Hence, $\bigcap_{\varepsilon>0} \Gamma(\varepsilon) \subseteq \Gamma$. Therefore, $\bigcap_{\varepsilon>0} \Gamma(\varepsilon)=\Gamma$. This completes the proof.

Next, we give an example to illustrate Proposition 3.1.

Example 3.1. Let $X=R, Z=R^{2}, K=\left\{\left(x_{1}, x_{2}\right) \in R^{2}:-4 \leq x_{1} \leq 4,-4 \leq x_{2} \leq 4\right\}, C=R_{+}^{2}=\left\{\left(x_{1}, x_{2}\right) \in\right.$ $\left.R^{2}: x_{1} \geq 0, x_{2} \geq 0\right\}, \omega=(10,10)$. Let $F, H: K \times K \rightarrow Z$ be defined as follows:

$$
F(x, y)=\left(x_{1}-\frac{y_{1}}{2}+3, x_{2}-\frac{y_{2}}{2}-10\right) \text { and } H(x, y)=\left(x_{1}+x_{2}-1, y_{1}-y_{2}-10\right) \text {. }
$$

It easy to see that

$$
x_{1} \geq\left(\frac{y_{1}}{2}-3\right)_{\max }=-1
$$

such that

$$
F(x, y) \notin-\operatorname{int} C .
$$

Hence,

$$
\Delta=\left\{\left(x_{1}, x_{2}\right) \in R^{2}:-1 \leq x_{1} \leq 4,-4 \leq x_{2} \leq 4\right\}
$$

Similarly, we have

$$
\Delta(\varepsilon)=\left\{\left(x_{1}, x_{2}\right) \in R^{2}:-1-10 \varepsilon \leq x_{1} \leq 4,-4 \leq x_{2} \leq 4\right\} .
$$

Since $H(x, y) \notin-\operatorname{int} C$, we conclude that $x_{1}+x_{2} \geq 1$. Therefore,

$$
\Gamma=\left\{\left(x_{1}, x_{2}\right) \in R^{2}: x_{1}+x_{2} \geq 1,-1 \leq x_{1} \leq 4,-4 \leq x_{2} \leq 4\right\} .
$$

Let $x, y \in \Delta$ be such that

$$
H(x, y)+\varepsilon \omega \notin-\operatorname{int} C .
$$

It follows that

$$
\Gamma(\varepsilon)=\left\{\left(x_{1}, x_{2}\right) \in R^{2}: x_{1}+x_{2} \geq 1-10 \varepsilon,-1 \leq x_{1} \leq 4,-4 \leq x_{2} \leq 4\right\} .
$$

Let $\varepsilon>0$ and $\varepsilon \rightarrow 0$. It is easy to obtain that $\bigcap_{\varepsilon>0} \Gamma(\varepsilon)=\Gamma$. 


\section{METRIC CHARACTERIZATIONS OF THE WELL-POSEDNESS}

In this section, we focus on the metric characterizations of the well-posedness for the BVEP. For this, we recall some basic concepts and known results.

Definition 4.1. [27] Let $A$ and $B$ be nonempty subsets of $X$. The Hausdorff distance $H(\cdot, \cdot)$ between $A$ and $B$ is define by

$$
H(A, B)=\max \{e(B, A), e(A, B)\},
$$

where $e(A, B)=\sup \{d(a, B): a \in A\}$ with $d(a, B)=\inf \{d(a, b): b \in B\}, d$ is a metric distance which is compatible with the topology of $X$.

Definition 4.2. [27] The Kuratowski measure of non-compactness of a set $A \subseteq X$ is defined by

$$
\alpha(A)=\inf \left\{\varepsilon>0: A \subset \cup_{i=1}^{n} A_{i}, \operatorname{diam} A_{i}<\varepsilon, i=1,2, \ldots, n\right\},
$$

where $\operatorname{diam}_{i}$ is the diameter of $A_{i}$ defined by

$$
\operatorname{diam}\left(A_{i}\right)=\sup \left\{d\left(x_{1}, x_{2}\right): x_{1}, x_{2} \in A_{i}\right\} .
$$

Under the suitable conditions, we can establish a metric characterization of generalized well-posedness for the BVEP by using the Kuratowski measure of non-compactness of the approximating solution sets.

Theorem 4.1. Let $X$ and $Z$ be Hausdorff topological vector spaces and let $K$ be a nonempty, closed and convex subset of $X$. Let $C \subset Z$ be a proper, closed, convex pointed cone, and let d be a metric which is compatible with the topology of $X$. Let $F, H: K \times K \rightarrow Z$ be two vector-valued bifunctions. Assume that all the following conditions are satisfied

(i) $X$ is complete;

(ii) for each $y \in K, x \mapsto H(x, y)$ is $C$-upper semicontinuous on $K$ in the first argument.

Then, the BVEP is generalized well-posed if and only if

$$
\Gamma(\varepsilon) \neq \emptyset, \forall \varepsilon>0 \text { and } \lim _{\varepsilon \rightarrow 0} \alpha(\Gamma(\varepsilon))=0 .
$$

Proof. Suppose that the BVEP is generalized well-posed. Then $\Gamma$ is compact. Let $\left(x_{n}\right)_{n \in N}$ be an approximating sequence. It has a subsequence converging to some points in $\Gamma$. For any $\varepsilon>0$, we have

$$
H(\Gamma(\varepsilon), \Gamma)=\max \{e(\Gamma(\varepsilon), \Gamma), e(\Gamma, \Gamma(\varepsilon))\}=e(\Gamma(\varepsilon), \Gamma) .
$$

Hence,

$$
\alpha(\Gamma(\varepsilon)) \leq 2 H(\Gamma(\varepsilon), \Gamma)+\alpha(\Gamma)=2 e(\Gamma(\varepsilon), \Gamma),
$$

where $\alpha(\Gamma)=0$ provide that $\Gamma$ is compact. Next, we show that

$$
e(\Gamma(\varepsilon), \Gamma) \rightarrow 0 \text {, as } \varepsilon \rightarrow 0 .
$$

Suppose that there exist $\rho>0, \varepsilon_{n}>0$ with $\varepsilon_{n} \rightarrow 0$ and $x_{n} \in \Gamma\left(\varepsilon_{n}\right)$ such that

$$
d\left(x_{n}, \Gamma\right) \geq \rho .
$$

Since $x_{n} \in \Gamma\left(\varepsilon_{n}\right),\left(x_{n}\right)_{n \in N}$ is an approximating solution sequence for the BVEP. By Definition 2.3, there exists a subsequence $\left(x_{n_{j}}\right)_{j \in N}$ of $\left(x_{n}\right)_{n \in N}$ converging to some element of $\Gamma$ such that $d\left(x_{n_{j}}, \Gamma\right) \rightarrow 0$ as $j \rightarrow+\infty$. This contradict (4.4). Therefore, $\lim _{\varepsilon \rightarrow 0} \alpha(\Gamma(\varepsilon))=0$ follows from (4.2) and (4.3). 
Conversely, we assume that (4.1) holds. From condition (ii), $\Gamma(\varepsilon)$ is closed for all $\varepsilon>0$. Let $\left(x_{n}\right)_{n \in N}$ be any approximating solution sequence. Then, there exists $\varepsilon_{n}>0$ with $\varepsilon_{n} \rightarrow 0$ such that

$$
H\left(x_{n}, y\right)+\varepsilon_{n} \omega \notin-\operatorname{int} C, \forall y \in \Delta
$$

and

$$
F\left(x_{n}, z\right)+\varepsilon_{n} \omega \notin-\operatorname{int} C, \forall z \in K .
$$

Hence, $x_{n} \in \Gamma\left(\varepsilon_{n}\right)$. Observe that $\Gamma=\cap_{\varepsilon>0} \Gamma(\varepsilon)$. From (4.1), one concludes from [27] that $\Gamma$ is nonempty, compact and

$$
e\left(\Gamma\left(\varepsilon_{n}\right), \Gamma\right)=H\left(\Gamma\left(\varepsilon_{n}\right), \Gamma\right) \rightarrow 0 \text { as } \varepsilon_{n} \rightarrow 0 .
$$

It follows that $d\left(x_{n}, \Gamma\right) \rightarrow 0$. Thus, there exists $u_{n} \in \Gamma$ such that $d\left(x_{n}, u_{n}\right) \rightarrow 0$. Since $\Gamma$ is compact, then there exists $\bar{x} \in \Gamma$ such that

$$
u_{n_{j}} \rightarrow \bar{x}
$$

where $\left(u_{n_{j}}\right)_{j \in N} \subset\left(u_{n}\right)_{n \in N}$. So, there exists $\left(x_{n_{j}}\right)_{j \in N} \subset\left(x_{n}\right)_{n \in N}$ such that $d\left(x_{n_{j}}, \bar{x}\right) \rightarrow 0$. Hence, the BVEP is generalized well-posed. This completes the proof.

\section{Acknowledgments}

The authors are grateful to the reviewers for useful suggestions which improve the contents of this paper. This paper was supported by the Basic and Advanced Research Project of Chongqing (cstc2016jcyjA0239) and the Graduate Student Innovation Project of Chongqing (CYS18137).

\section{REFERENCES}

[1] J. Hadamard, Sur les problemes aux derivees partielles et leur signification physique, Princeton Univ. Bull. 13 (1902), 49-52.

[2] A. N.Tikhonov, On the stability of the functional optimization problem, USSR J.Comput. Math. Phys. 6 (1966), 28-33.

[3] E.S. Levitin, B.T. Polyak, Convergence of minimizing sequences in conditional extremum problems, Sov. Math. Dokl. 7 (1966), 764-767.

[4] L.J. Kettner, S. Deng, On well-posedness and Hausdorff convergence of solution sets of vector optimization problems, J. Optim. Theory Appl. 153 (2012), 619-632.

[5] X.X. Huang, X.Q. Yang, Levitin-Polyak well-posedness of constrained vector optimization problems, J. Glob. Optim. 37 (2007), 287-304.

[6] G.P. Crespi, A. Guerraggio, M. Rocca, Well-posedness in vector optimization problems and vector variational inequalities, J. Optim. Theory Appl. 132 (2007), 213-226.

[7] J. Yu, H. Yang, C. Yu, Well-posed Ky Fan's point, quasi-variational inequality and nash equilibrium problems, Nonlinear Anal. 66 (2007), 777-790.

[8] M. Margiocco, F. Patrone, L.P. Chicco, Metric characterizations of Tikhonov well-posedness in value, J. Optim. Theory Appl. 100 (1999), 377-387.

[9] L.Q. Anh, D.V. Hien, On well-posedness for parametric vector quasiequilibrium problems with moving cones, Appl. Math. 61 (2016), 651-668.

[10] M.H. Li, S.J. Li, W.Y. Zhang, Levitin-polyak well-posedness of generalized vector quasi-equilibrium problems, J. Ind. Manag. Optim. 5 (2009) ,683-696.

[11] K. Kimura, Y.C. Liou, S.Y. Wu, J.C. Yao, Well-posedness for parametric vector equilibrium problems with applications, J. Ind. Manag. Optim. 4 (2008), 313-327. 
[12] S.J. Li, M.H. Li, Levitin-Polyak well-posedness of vector equilibrium problems, Math. Methods Oper. Res. 69 (2009), 125-140.

[13] T. Shitkovskaya, J. Han, D.S. Kim, Generalized approximate solutions for multiobjective optimization with infinitely many criteria, Appl. Anal. Optim. 1 (2017), 401-410.

[14] X. Qin, J.C. Yao, Projection splitting algorithms for nonself operators, J. Nonlinear Convex Anal. 18 (2017), 925-935.

[15] A. Moudafi, Proximal methods for a class of bilevel monotone equilibrium problems, J. Global Optim. 47 (2010), $287-292$.

[16] X.P. Ding, Y.C. Liou, J.C. Yao, Existence and algorithms for bilevel generalized mixed equilibrium problems in Banach spaces, J. Global Optim. 53 (2012), 331-346.

[17] J.W. Chen, Z.P. Wan, Y.Z. Zou, Bilevel invex equilibrium problems with applications, Optim. Lett. 8 (2014), $447-461$.

[18] L.Q. Anh, P.Q. Khanh, D.T.M. Van, Well-posedness under relaxed semicontinuity for bilevel equilibrium and optimization problems with equilibrium constrains, J. Optim. Theory Appl. 153 (2014), 42-59.

[19] J.W. Chen, Y.J. Cho, Z.P. Wan, The existence of solutions and well-posedness for bilevel mixed equilibrium problems in Banach spaces, Taiwanese J. Math. 17 ( 2013), 725-748.

[20] P.Q. Khanh, S. Plubtieng, K. Sombut, LP well-posedness for bilevel vector equilibrium and optimization problems with equilibrium constains. Abstr. Appl. Anal. 2014 (2014), Article ID 792984.

[21] O. Chadli, Q. H. Ansari, S. Al-Homidan, Existence of solutions and algorithms for bilevel vector equilibrium problems: an auxiliary principle technique, J. Optim. Theory Appl. 172 (2017), 726-758.

[22] L.Q. Anh, N.V. Hung, Stability of solution mappings for parametric bilevel vector equilibrium problems, Comput. Appl. Math. 37 (2018), 1537-1549.

[23] L.Q. Anh, N.V. Hung, Levitin-Polyak well-posedness for strong bilevel vector equilibrium problems and applications to traffic network problems with equilibrium constraints, Positivity. 22 (2018), 1223-1239.

[24] T. Tanaka, Generalized semicontinuity and existence theorems for cone saddle point, Appl. Math. Optim. 36 (1997),313322.

[25] K. Kimura, J.C. Yao, Sensitivity analysis of solution mappings of parametric vector quasi-equilibrium problems, J. Global Optim. 41 (2008), 187-202.

[26] J.W. Chen, X.X. Ju, Y.C. Liou, C.F. Wen, Existence of solutions and well-posedness for bilevel vector equilibrium problems, J. Nonlinear Convex. Anal. 19 (2018), 2203-2217.

[27] K. Kuratowski, Topology,vols 1 and 2, Academic Press, New York, NY, 1968. 